\title{
REFLECTIONS ON EDITING MOORE'S NOTES IN WITTGENSTEIN: LECTURES, CAMBRIDGE 1930-1933
}

\begin{abstract}
The essay begins by briefly reviewing the complex history of the collaborative long-distance editing work that led to the publication of Wittgenstein: Lectures, Cambridge 1930-1933 (Cambridge UP, 2016). It then turns to a discussion of the rationale for the innovative editorial policies we ultimately developed and implemented, and some of the broader methodological issues that they raise.
\end{abstract}

In August 2009, while we were taking part in the annual Kirchberg Wittgenstein Symposium, Josef Rothhaupt asked Brian Rogers and myself to work with him on an edition of Moore's notes of Wittgenstein's lectures. I contacted Thomas Baldwin, Moore's literary trustee, to ask for permission to edit the notes. Baldwin informed us that he had already given Gabriel Citron permission to produce an edition of the final set of notes, those from the May 1933 term, and he suggested that we explore the possibility of working together with Citron on an edition of the full set of notes. After some discussion, Citron agreed to work with us toward the larger project, and Rothhaupt graciously withdrew. ${ }^{1}$ As Citron had already prepared a first draft transcription of the May 1933 lectures, the initial stage of our collaboration was a matter of Rogers and myself checking his transcription against low-resolution photocopies of the originals, and of the three of us settling on preliminary protocols for jointly editing the material long-distance. That revised draft of the May 1933 lecture notes then served as the basis for a book proposal for Cambridge University Press, which was accepted in August 2010. Cambridge University Press was the ideal venue for this project for a number of reasons. First, I had worked with the Press for some time as an editor and author, and so had a good working relationship with Hilary Gaskin, their Philosophy editor. She was supportive of our plans for a scholarly edition of the Moore

1 I want to take this opportunity to thank Brian Rogers and Gabriel Citron for making such an extraordinarily productive collaboration possible. I would also like to thank Eran Guter for discussing how best to edit Moore's notes of Wittgenstein's lectures with me while I was working on this paper, and Jim Levine and Bernie Linsky for a discussion of how best to edit Moore's notes of Russell's lectures. 
manuscripts, even when it involved a number of substantial departures from the Press's usual typographic and editing conventions. In addition, the close relationship between Cambridge University Library, the owner of the manuscripts, and Cambridge University Press, meant that we could use images of the manuscripts without any of the charges that would have been levied on an edition at another press. This proved to be crucial when we decided to use over fifty images of each of Moore's drawings in the book, and to produce a website containing high-resolution images of every page of the notes. The first draft of the book contract in my files is dated February 2011, with a delivery date of January 2012; the version we finally signed was dated October 2012, with a September 2014 delivery date. The book manuscript was sent to the Press in December 2014, and was published in November 2016.

As this very brief timeline already suggests, we encountered more difficulties in the process of editing the book than we had initially anticipated. Some of them, such as the challenges involved in long-distance collaboration between three people, working on reproductions of a complex and sometimes barely legible manuscript, were evident from the start. Those 'known unknowns', turned out to be much less trouble than one might have reasonably expected. In large part, that was thanks to exceptional patience and good will on the part of everyone involved. It was also due to rapid technological progress in photoreproduction, and a generous Franklin Research Grant from the American Philosophical Society. That grant enabled us to replace the low-resolution black and white scans that were available when we began our collaboration, digital images that were every bit as fuzzy and illegible as the output of an average library photocopier in the 1970s. They were replaced by high-resolution color scans, so detailed that one could easily make out some details onscreen that were barely visible when looking at the original manuscript in good light.

It was the 'unknown unknowns', the difficulties that we did not anticipate when we first planned our collaboration, that ultimately turned out to be the most challenging and demanding. For the most part, those difficulties arose from the overly optimistic expectation that we could easily build on the work that we had done on the substantial sample chapter containing the last term's notes and straightforwardly apply it to the project of editing the notes from the other terms. Now that we had established a set of editing protocols in the course of constructing our writing sample, we thought we simply needed to go on in the same way, perhaps with some minor modifications and extensions, to the remainder of the manuscript text. However, Moore's notes from the May 1933 term turned out to be, in a number of ways, unrepresentative of the notes from the previous eight terms. When examined more closely, those manuscripts led us to a number of problems that we had not anticipated. First, because Moore gradually became more adept at 
taking notes in Wittgenstein's lectures, and because Wittgenstein gradually became a more experienced lecturer, the last set of notes were much more polished than the earliest ones, which were often far more telegraphic and sometimes clearly incomplete. Second, it only gradually became clear to us how difficult it would be to find a principled way of separating out the words Moore wrote down as lecture notes from his subsequent comments and his own editorial and philosophical responses. While his notes were frequently very detailed, they were sometimes, and especially in the first few terms' notes, rather telegraphic, and often overlaid with his own second thoughts about the notes themselves. Although some of those additions were probably made while Moore was still present in the class in question, or very shortly afterwards, it was clear that most of them dated from his own research some twenty years later, when he was writing his articles for Mind on the lectures. Third, the May 1933 notes contained only three small drawings, and made very little use of mathematical and logical notation. There were over fifty more diagrams and drawings in the notes for the previous terms, and some of those lectures made extensive use of symbolism, which Moore had clearly tried to transcribe with great fidelity, but had sometimes written down in ways that were obviously mistaken. Thus, we had to arrive at a consistent and appropriate way of editing not only the words on the page, but also the logical and mathematical symbolism, and the dozens of diagrams, almost all of which were clearly an integral part of the lectures.

While the principal editorial policies we ultimately adopted are concisely stated in the final part of our editors' introduction (Stern, Citron, and Rogers, xlix-lvi), our reasons for adopting them are only briefly summarized there. However, the underlying rationale that guided those decisions can be stated in a couple of sentences. All three of us agreed, from the beginning of our collaboration, that we wanted, as best we could, to give the reader access to what Moore wrote down at the time. However, we also wanted to find a way of combining a high level of fidelity to the source manuscript with a clear and easily legible text.

In the opening of the final section of our editorial introduction, we summed up the methods we ultimately embraced in the following words:

Our main objectives in editing Moore's notes were both to provide a text that makes Wittgenstein's lectures accessible and to reproduce faithfully what Moore actually wrote down. Because Moore's lecture notes are so thorough and conscientious, they deserve to be published in full, with a minimum of editorial intervention or revision. However, reproducing every mark on the notebook pages would have resulted in an inaccessible and distracting text, hindering readers from engaging with the content of the lectures. We have therefore amended the text only when the benefits of doing so outweighed the primary value of providing an exact reproduction. We have also employed a minimal 
editorial apparatus in this volume in order to minimize distractions from the content of the lectures. (Stern, Rogers and Citron 2016, xlix)

In the remainder of that section, we set out our general editorial policies and illustrated them by discussing their application to examples drawn from four sample facsimile manuscript pages. However, that eight-page discussion, the capstone of over fifty pages of editorial introduction, was of necessity no more than a brief summary.

Our programmatic and seemingly simple aim of producing a consistently edited and accessible text on the principle of fidelity to what Moore wrote down at the time, ultimately led us into a protracted and intricate discussion of how to put that principle into practice. In part, this was simply a matter of working out how to handle any number of unexpected and initially overlooked details, the particular challenges we faced in doing justice to the specific character of Moore's contemporaneous notes. However, there was also a shared pattern to many of these decisions about how to handle any number of specific matters of editorial policy. For our overarching aim was to build on the complementary strengths of the two main editorial approaches taken in previous editions of Wittgenstein's work. The first generation of Wittgenstein's editors, including his literary executors, and previous editors of his lecture notes, concentrated on providing a clear, uncluttered and easily accessible text. The next generation of editors have mostly followed the example of the much more scholarly and systematic approach pioneered by the Bergen Wittgenstein Archive which offers much greater fidelity to the source text, but on the other hand does so in a much less easily accessible form. In the early 1990s, I became very interested in the Bergen Wittgenstein Archive's work on a digital edition of Wittgenstein's Nachlass, and I first visited the Archive in 1992. Since then, their work has not only been a crucial resource for my research, but has also led me to think about the theory and practice of Wittgenstein editing in particular, and the philosophical issues raised by the practice of editing philosophy (See Stern 1994, 1996, 2008, 2010).

We wanted, as much as possible, to produce an edition that was as accessible and uncluttered as the first editions of Wittgenstein's lectures, yet also as careful about getting the words on the page right as the current generation of Wittgenstein editors. In other words, the editorial approach that we envisaged aimed at a synthesis of the seemingly diametrically opposed approaches that had guided previous editors of Wittgenstein's work. On the one hand, we admired the clear, uncluttered and easily accessible editions produced by his literary executors, and previous editors of notes taken at his lectures (Wittgenstein, 1966, 1976, 1979, 1979a, 1980, 1988). However, that first generation of editors had often made questionable decisions about how to present that material, including decisions about what to include and what to leave out, at times rearranging sketchy notes and filling in gaps 
when they thought it necessary, and they usually said little or nothing about those choices and their reasons for making them. The previous editions of student notes of Wittgenstein's lectures from the early 1930s (Wittgenstein, 1979a, 1980) were a particularly clear case of this kind of editorial freehandedness. Probably the best-known and most controversial example of this way of editing Wittgenstein was Rhees' editing of the Philosophical Grammar (Wittgenstein 1974; for further discussion, see: Kenny 1976, Hintikka 1991 and 2005, Stern 1996).

On the other hand, we were also impressed by the much more scholarly and systematic approach pioneered by the Bergen Wittgenstein Archive (Wittgenstein 2000, 2009). That online editing and the published texts it inspired, such as the 'Scholar's Edition' of The Big Typescript (Wittgenstein 2005), pioneered a much more transparent and explicitly stated approach to Wittgenstein editing, and achieved much greater consistency and scholarly fidelity to the source text. However, these virtues came at a high price, for showing all of the complex changes made by the author involved introducing an intricate editorial apparatus that could be quite opaque and forbidding to the casual reader (see Wittgenstein 2005 viii-ix for a list of the abbreviations and other conventions used there, for instance.)

One compromise that is easily available online is to provide multiple versions of the same text. For instance, the Bergen Electronic Edition (2000) of Wittgenstein's papers provides not only a 'diplomatic' edition that shows a very high level of editorial detail, but also a much simpler 'normalized' version which only shows the final outcome of the various stages of revision represented in the 'diplomatic' version, plus photographic facsimiles. The Wittgenstein Source Bergen Nachlass Edition (2009) goes a step further, allowing the reader to tailor a customized normalized version by choosing from an extended menu of presentation options. This approach allows the scholar to closely investigate the textual details by working with the facsimiles and the diplomatic transcription, while a casual reader can simply browse the normalized version.

Our response to the challenge of working out how to employ a minimal editorial apparatus yet maximize fidelity to Moore's original notes turned on the decision that while we would start by drawing up a diplomatic transcription of the notes, the text of our published edition would be a consistently normalized version of that transcription. Instead of showing all the details of the various changes that Moore had made, we would instead regard his contemporaneous changes (and his later disambiguation of his sometimes barely legible writing, and occasional correction of obvious mistakes) as instructions for producing a much more legible normalized text. Because we did this in a systematic and consistent manner, without any surreptitious additions or changes on our part, we could thus provide access to what Moore wrote, not our own reconstruction of his words. 
Nevertheless, as part of that normalization process, we did decide to correct Moore's choice of words in a few clearly defined categories of straightforward mistakes where a literal transcription could only have caused confusion. In particular, we silently corrected the occasional misspelled words and smaller errors in matters of logical notation. We also filled in Moore's many abbreviations of frequently used words, and provided a glossary of his abbreviations at the end of the book, judging that this would make the text much easier to take in, and would not deprive the reader of any useful information. However, we almost entirely avoided inserting words or punctuation of our own, and on those occasions when we thought something missing had to be included, we used a distinctive grey font.

A number of closely related issues arose from the fact that the documents we were editing were not consistently formatted, and so the use of spacing and the organization of words and sentences on the page was often somewhat idiosyncratic, yet was also sometimes semantically significant. For instance, Moore frequently used very large spaces before and after a word or phrase as mentioning device. If we had simply kept the extra spacing, many readers would have missed its significance. Adding an explanatory footnote would have been repetitive and cumbersome, but omitting the spacing altogether would have been seriously misleading. This is a good example of a case where grey font enabled us to solve this problem elegantly: we replaced the spaces by a pair of quotation marks in grey.

We knew that dedicated scholars would want to check up on our decisions on these and similar matters, and so we provided access to the facsimiles we used on the Wittgenstein Source website (Stern, Citron, and Rogers, 2015). In order to make it easy for readers of our edition to find the corresponding page of the source manuscript on the web, we had to provide manuscript page numbers in the margin of each page of our printed edition. This use of manuscript page numbers, in turn, made it easier for us to explain various problematic features of the text, such as a few exceptional places where we had to reorganize text because Moore had included arrows connecting material on different pages.

A solitary editor, or even a pair of editors working in close proximity, might well be able to rely on memory and habit to ensure that they went on in the same way. However, as the three of us were thousands of miles apart and almost entirely reliant on email as our primary form of communication, we soon realized that we had to come up with detailed editorial protocols if we were to remain on the same page, so to speak. As a result, we spent many months negotiating the drafting of a master document setting out these editorial procedures and policies, which gradually became increasingly intricate and complex.

We divided the task of editing the manuscript notes into two distinct phases. First, we aimed to produce 'diplomatic' editions of each manuscript. 
In this stage of the process, the aim was to transcribe every potentially semantically significant aspect of the notes, with only minimal editorial intervention. For instance, we recorded all of Moore's words, including his later comments and editorial mark-up from the 1950s, and all crossed out words were included in 'strikethrough' font. At this stage, we did do some initial editing of the text, such as omitting pages that were clearly authored by Moore, including his indexes at the end or beginning of the notes, and some pages consisting entirely of his own work that were clearly separate from the lecture notes.

The work of diplomatic transcription was shared out roughly equally among the three of us, and the initial stage of diplomatic transcription, while time-consuming in terms of the work needed to produce a first draft, went relatively quickly and smoothly. It was only as we began the further task of checking each other's work that we realized how many of our construals of individual words needed further review, and how many policy questions about how to handle difficult and unexpected cases had arisen. Indeed, over an extended period of time working on hundreds of pages of transcriptions, it is extraordinarily challenging for any one person to simply keep track of those policies and be sure that one has applied them appropriately in each and every case. In practice, the precise details of our principles of transcription proved to be a work in progress and gradually evolved over time.

Matters became even more complicated in the next stage of the work, as we had to settle on policies to be implemented in turning the initial 'diplomatic' transcriptions into the 'normalized' product that we would ultimately use in the published edition of the notes. We remained consistently committed to the broad outline of the normalization policies described above. We would produce an edition of Moore's contemporaneous lecture notes, omitting his own later writing from the main text, but including the most interesting later comments and remarks in footnotes. We would not silently add our own words and punctuation to fill in the gaps and organize his fragmentary notes. However, we still had to deal with a large number of more specific decisions about how best to implement that strategy, all of which then had to be consistently applied by each editor. Despite these carefully formulated plans, there were often unexpected cases that required us to adjust our policies. However, there eventually came a point at which we couldn't adjust them any further, if only because doing so would require systematically checking the entire text to make sure that the change was carried out consistently. In those cases we had to make exceptions, and if the departure seemed substantial or significant, we added an explanatory footnote explaining what we had done.

It was not easy to decide what kinds of information to include in the footnotes, and how detailed they should be. Many scholars and researchers favor the inclusion of many detailed footnotes, believing that the extra information can only be helpful, and that the casual reader is always free 
to ignore them. We did include many of Moore's later responses to what Wittgenstein said, and such matters as small variants to the wording, etc., in our footnotes until a quite late stage in the process. However, we were ultimately persuaded to substantially reduce the number of such footnotes by a reader, who told us that he found the sheer number of relatively inconsequential footnotes distracting, not only because it made it harder to read the text itself, but also because the small number of really interesting footnotes were lost in the welter of boring ones.

In the end, we decided to restrict ourselves to a few relatively simple categories of footnotes. We included references for both explicit and implicit citations of works by others whenever possible, and also for clear allusions. However, we limited ourselves to only a very few cross-references to Wittgenstein's other writings, providing them only where we considered a parallel passage to be essential, or at least very helpful, for understanding particular passages. This was largely a pragmatic decision to avoid taking on what would have been a gigantic task if it were done thoroughly, as there are so many parallels and connections between the lectures and Wittgenstein's other writings.

A few particularly difficult editorial questions were deferred at each stage in the editing process, in the hope that they would be easier to resolve later on. While we were working on the diplomatic transcriptions, there were thousands of words that were either wholly or partially illegible, or at least sufficiently unclear that they were marked as doubtful. As time went by, we became increasingly skilled at recognizing Moore's handwriting, and his characteristic ways of writing certain letters. However, at the end of spring 2016, some time after we had circulated an almost-final transcription of the complete set of lectures, we still had a list of about a hundred or so particularly difficult words. I was able to resolve a number of them by returning to Cambridge University Library and carefully examining the text. At this stage, we no longer had any completely illegible words; rather, we were looking at words where we could only construe some of the letters, and several different guesses as to how to complete the word all seemed quite plausible. This made it possible for us to identify those words by a new strategy: comparing the barely legible letters with instances of similar letters elsewhere in the Moore manuscripts. For we realized that our transcription could be used as a database of successful identifications of the various sequences of letters Moore had written down, and so we could search it for each of the candidate construals of a given illegible word, and could then compare it with the facsimiles of words that we had successfully construed. As an artificial but illustrative example, imagine that we were unable to work out whether a given word which began with 'th' was a token of 'this' or 'that'. In most cases, a final 'is' and a final 'at' in Moore's hand are easy to distinguish: there is almost always a dot on the ' $i$ ' and a horizontal line on the ' $t$ '. But if both of those 
markers were missing, and the end of the word was scribbled in a hurry, we would then bring up other instances of scrawled tokens of 'this' and 'that', and compare the illegible word with those two sets of patterns. In every case but one in which the 'semantic' approach-looking for meaningful words-failed to provide a resolution, this 'syntactic' technique-looking for recognizable tokens of this letter after that one-was successful. In the end, the only word that we were unable to construe by this method was a singleton, an obscure name that occurs only once. It clearly begins with an ' $\mathrm{N}$ ', and probably ends in 'benites' or 'lenites', with approximately three letters in between (Wittgenstein 2016, 361 9:44).

One of the main aims of this paper is to provide some insight into the lengthy process of deliberation that led us from our guiding principles to the particular implementation that we ultimately chose. I hope this account will be of use not only to readers of our edition of Moore's notes, but also to those who are considering how best to edit similar manuscripts in the future.

\section{References}

Hintikka, Jaakko 1991. 'An Impatient Man and His Papers', Synthese, 87: 183201.

Kenny, Anthony 1976. 'From the Big Typescript to the Philosophical Grammar', Acta Philosophica Fennica, 28: 41-53. [Reprinted in A. Kenny, The Legacy of Wittgenstein, 1984, 24-37. Oxford: Blackwell.]

Kenny, Anthony 2005. 'A Brief History of Wittgenstein Editing. In A. Pichler and S. Säätelä (eds.), Wittgenstein: The Philosopher and his Works, 341-55. Bergen: Wittgenstein Archives at the University of Bergen.

Sluga, Hans and David Stern (eds.) 1996. The Cambridge Companion to Wittgenstein. Cambridge: Cambridge University Press.

Stern, David G. 1994. 'The Wittgenstein Papers as Text and Hypertext: Cambridge, Bergen, and beyond'. In Kjell Johannessen, Rolf Larsen and Knut Olav Amas (eds.), Wittgenstein and Norway, 251-273. Oslo: Solum Press.

Stern, David G. 1996. 'The Availability of Wittgenstein's Philosophy'. In Sluga and Stern 1996, 442-476.

Stern, David G. 2008. 'Digital Wittgenstein Scholarship: Past, Present and Future.' In Alois Pichler and Herbert Hrachovec (eds.), Wittgenstein and the Philosophy of Information: Proceedings of the 30th International Wittgenstein Symposium, Vol. 1, 223-238. Frankfurt: Ontos Verlag.

Stern, David G. 2010. 'The Bergen Electronic Edition of Wittgenstein's Nachlass.' European Journal of Philosophy, 18: 455-467. 
Stern, David G, Brian Rogers and Gabriel Citron (eds.) 2015. Wittgenstein Source Facsimile Edition of Moore's Notes of Wittgenstein's Lectures (WS$M W N)$. Edited by David Stern, Brian Rogers, and Gabriel Citron. http:// www.wittgensteinsource.org/

Stern, David G, Brian Rogers and Gabriel Citron 2017. 'Editorial Introduction'. In Wittgenstein 2016, xi-lx.

Wittgenstein, Ludwig 1966. Lectures and Conversations on Aesthetics, Psychology and Religious Belief. Edited by Cyril Barrett. Berkeley: University of California Press.

Wittgenstein, Ludwig 1974. Philosophical Grammar. English translation by Anthony Kenny. Oxford: Blackwell. [First published in 1969 as Philosophische Grammatik, German text only, edited by Rush Rhees. Oxford: Blackwell.]

Wittgenstein, Ludwig 1976. Wittgenstein's Lectures on the Foundations of Mathematics, Cambridge 1939. Edited by Cora Diamond. Ithaca: Cornell University Press.

Wittgenstein, Ludwig 1979. Wittgenstein and the Vienna Circle. Edited by B. McGuinness, translated by J. Schulte and B. McGuinness. Oxford: Blackwell. [First published in German in 1967].

Wittgenstein, Ludwig 1979a. Wittgenstein's Lectures, Cambridge 1932-1935: From the Notes of Alice Ambrose and Margaret MacDonald. Edited by Alice Ambrose. Chicago: University of Chicago Press.

Wittgenstein, Ludwig 1980. Wittgenstein's Lectures: Cambridge, 1930-1932: From the Notes of John King and Desmond Lee. Edited by Desmond Lee. Chicago: University of Chicago Press.

Wittgenstein, Ludwig 1988. Wittgenstein's Lectures on Philosophical Psychology: 1946-47. Edited by Peter Geach. Chicago: University of Chicago Press.

Wittgenstein, Ludwig 2000. Wittgenstein's Nachlass: The Bergen Electronic Edition. Oxford: Oxford University Press.

Wittgenstein, Ludwig 2005. The Big Typescript: TS 213. Edited and translated by C. Grant Luckhardt and Maximilian A.E. Aue. Oxford: Blackwell.

Wittgenstein, Ludwig 2009. Wittgenstein Source Bergen Nachlass Edition. Edited by the Wittgenstein Archives at the University of Bergen under the direction of Alois Pichler. Bergen: WAB. http://www.wittgensteinsource.org/

Wittgenstein, Ludwig 2016. Wittgenstein: Lectures, Cambridge 1930-1933: From the Notes of G. E Moore. Edited by David Stern, Brian Rogers, and Gabriel Citron. Cambridge: Cambridge University Press. 